\title{
A SMOOTH ACTION ON A SPHERE WITH ONE FIXED POINT
}

\author{
BY ELLIOTT STEIN
}

Communicated by Glen C. Bredon, August 27, 1975

In this note, we outline the construction of a smooth action of the finite group $\operatorname{SL}\left(2, Z_{5}\right)$ on $S^{7}$ with exactly one fixed point. Along with some related examples given below, this is the first partial result of a positive nature on the problem of deciding which compact Lie groups can act smoothly on some sphere with one fixed point.

Let $S^{\mathbf{3}}$ denote the multiplicative group of unit quaternions, and let $G \subset S^{\mathbf{3}}$ be a subgroup isomorphic to $\operatorname{SL}\left(2, Z_{5}\right)$, the binary icosahedral group. The space of right cosets, $S^{3} / G$, is an integral homology sphere, denoted $\Sigma^{3}$. As pointed out in [2], the natural action of $G$ on $\Sigma^{3}$, although not effective, has exactly one fixed point. We denote this action by $\lambda$. Let $\mu$ denote the action of $G$ on $D^{4}$ given by quaternionic multiplication; this action is free away from the origin. The diagonal action, $\lambda \times \mu$, on $\Sigma^{3} \times D^{4}$ has exactly one fixed point and is free on the boundary.

Now let $f: M^{4} \rightarrow D^{4}$ denote the collapsing map from the four-dimensional Milnor manifold to a disk. After covering $f$ with a map of stable normal bundles, we obtain a normal map whose surgery obstruction generates the Wall group, $L_{0}(e)$.

LEMMA. The surgery obstruction, in $L_{3}(G)$, of the normal map $f \times$ identity: $M^{4} \times \Sigma^{3} \rightarrow D^{4} \times \Sigma^{3}$ is zero.

Based on this Lemma, the construction is completed as follows. There is a normal cobordism, with the boundary fixed, from $f \times$ identity to $F: W^{7} \rightarrow D^{4}$ $\times \Sigma^{3}$, with $F$ a homotopy equivalence. There is a free action of $G$ on $\widetilde{W}$, the universal cover of $W$, by covering translations. It is now easy to verify: (1) There is an equivariant diffeomorphism, $\varphi$, from the boundary of $\widetilde{W}$ to the restriction of $\lambda \times \mu$ to $\Sigma^{3} \times S^{3}$; (2) The $G$-manifold obtained by gluing $\widetilde{W}$ to $\Sigma^{3} \times D^{4}$ by $\varphi$ is a homotopy sphere. Now by using a slight variation of "equivariant connected sum", this action can be modified, away from the single fixed point, so that the ambient manifold is diffeomorphic to $S^{7}$.

The proof of the Lemma is quite technical and can only be outlined here. First, the induction theorem of Dress [1] is used to reduce the Lemma to the computation of some surgery obstructions with simpler fundamental groups.

AMS (MOS) subject classifications (1970). Primary 57E25, 57D65. 
These surgery problems, obtained as coverings of the normal map in the Lemma, are solved geometrically by doing surgery explicitly below the middle dimension, and then constructing embedded spheres in the middle dimension so that surgery yields a homotopy equivalence. No specific information about the Wall groups is necessary for the proof.

The construction above can be extended slightly using a recent theorem of Wall [4] which reduces the Lemma to a similar statement involving the Sylow 2-subgroup of $G$. This enables us to prove the following

Proposition. Let $G=\operatorname{SL}\left(2, Z_{5}\right)$ and let $H$ denote either the trivial group or a cyclic group whose order is relatively prime to $|G|=120$. Then there exists a smooth action of the group $G \times H$ on $S^{7}$ with exactly one fixed point.

Acknowledgement. These results are essentially those obtained in [3]. I am grateful to Wu-chung Hsiang for the encouragement and advice that made the completion of this work possible.

\section{REFERENCES}

1. A. Dress, Induction and structure theorems for Grothendieck and Witt rings of orthogonal representations of finite groups, Bull. Amer. Math. Soc. 79 (1973), 741-745. MR 49 \#7345.

2. E. E. Floyd and R. W. Richardson, An action of a finite group on an n-cell without stationary points, Bull. Amer. Math. Soc. 65 (1959), 73-76. MR 20 \#7276.

3. E. Stein, $A$ smooth action on a sphere with one fixed point, Thesis, Princeton University, 1975.

4. C. T. C. Wall, Formulae for surgery obstructions (to appear).

DEPARTMENT OF MATHEMATICS, PRINCETON UNIVERSITY, PRINCETON, NEW JERSEY 08540

Current address: Department of Mathematics, University of Chicago, Chicago, Illinois 60637 